\title{
Absceso hepático piógeno en pediatría: experiencia en un centro pediátrico de referencia
}

\author{
Carolina Carballo, Claudia Cazes, Mariana Matsuda, M. Laura Praino, Noemí Rivas y Eduardo López
}

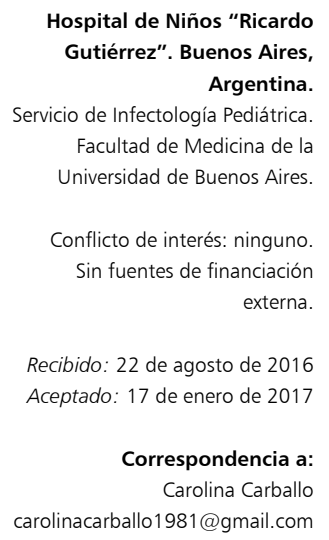

Hospital de Niños "Ricardo Gutiérrez". Buenos Aires, Argentina.

Servicio de Infectología Pediátrica.

Facultad de Medicina de la Universidad de Buenos Aires.

Conflicto de interés: ninguno Sin fuentes de financiación externa.

Recibido: 22 de agosto de 2016 Aceptado: 17 de enero de 2017

Correspondencia a: Carolina Carballo carolinacarballo1981@gmail.com

\section{Pyogenic liver abscess in children: clinical experience in a pediatric reference center}

Introduction: Liver abscess is a serious and an uncommon pediatric disease. Objective and Method: Description of 20 pediatric patients with liver abscess admitted in "Ricardo Gutierrez" Children's Hospital, a Tertiary Reference Center of Buenos Aires, between 2009 and 2015. Results: The hospitalization rate was 35/100.000 admissions. Median age was 5 years old, male/female ratio 4/1. Five patients $(25 \%)$ had predisposing factors. Median days at diagnosis were 12 . Features: Fever $100 \%$, prolonged fever $60 \%$, abdominal pain $50 \%$. The majority had leukocytosis and elevated CRP (median $160 \mathrm{mg} / \mathrm{L}$ ). Liver enzymes were elevated in only $40 \%$ of patients. Ultrasonography detected $90 \%$. A single abscess was observed in $60 \%$; localized at right hepatic lobe, $65 \%$. Purulent material was positive in $12 / 17(70 \%)$ and bacteremia was present in 3/20 (15\%). Wide empirical antibiotic therapy was used. Treatment shift was needed in four patients. The median days of intravenous antibiotics were 30 and the median of total treatment were 53. Surgical procedures were performed in 13/20 (65\%). Conclusion: Community-acquired methicillin resistant Staphylococcus aureus (CA-MRSA) was the most frequent pathogen, especially in healthy children that associated skin or respiratory illness. Appropriate antibiotic treatment and eventual drainage allows good outcome without sequela or deaths.

Key words: Pyogenic liver abscess, liver abscess, abdominal abscess.

Palabras clave: Absceso hepático piógeno, absceso hepático, absceso abdominal.

\section{Introducción}

$\mathrm{E}$ 1 absceso hepático $(\mathrm{AH})$ es una patología poco frecuente en la niñez ${ }^{1,2}$. En países en desarrollo, su mayor incidencia está asociada a malnutrición o parasitosis, que se describen como factores predisponentes ${ }^{1}$.

En el AH piógeno, Staphylococcus aureus y bacilos gramnegativos entéricos, representan $80 \%$ de las etiologías bacterianas. Los signos y síntomas más frecuentes son fiebre y dolor abdominal, manifestaciones inespecíficas que pueden corresponder a otras entidades nosológicas en niños, lo que dificulta el diagnóstico. La ecografía y la TC abdominal son los métodos de elección para confirmar el diagnóstico. Habitualmente los AH son únicos, localizados en el lóbulo derecho. El tratamiento se basa en el tratamiento antimicrobiano prolongado, con o sin drenaje quirúrgico. Aunque con baja mortalidad son causa de internaciones prolongadas debido a la administración de antimicrobianos intravenosos ${ }^{1,2}$.

Existen escasos datos sobre las características clínicas, epidemiológicas y microbiológicas de los AH piógenos en niños de nuestra región. En este contexto, el objetivo de nuestro estudio fue revisar los aspectos clínicos, etiológicos, de diagnóstico y tratamiento en la población estudiada.

\section{Materiales y Métodos}

Se realizó un estudio descriptivo, retrospectivo. Se revisaron las historias clínicas de niños menores de 18 años con diagnóstico al egreso de $\mathrm{AH}$ internados entre los años 2009 y 2015 en el Hospital de Niños "Dr. Ricardo Gutiérrez" de Buenos Aires, Argentina.

Microbiología: Para los cultivos se utilizó el sistema Bact-alert (Biomerieux, Marcy, L’Étoile, Francia). Se identificó a las especies $S$. aureus, Escherichia coli, Klebsiella pneumoniae y Streptococcus viridans por pruebas microbiológicas habituales. La susceptibilidad a meticilina se determinó mediante pruebas de difusión con discos de oxacilina de 1 ug y de cefoxitina de 30 ug, en medio agar de Mueller Hinton (MHA) con incubación a $37^{\circ} \mathrm{C}$ durante $24 \mathrm{~h}$. La detección de halos menores de $20 \mathrm{~mm}$ se consideró como resistente. Se realizó detección selectiva en placa de MHA con $4 \%$ de cloruro de sodio y 6 $\mathrm{ug} / \mathrm{ml}$ de oxacilina, con inóculo de 100.000 ufe. Toda cepa que se desarrolló fue considerada resistente a meticilina según normas del Instituto de Estándares Clínicos y de Laboratorio (CLSI, por su sigla en inglés) de los E.U.A. ${ }^{3}$

Se utilizaron los criterios diagnósticos de sepsis en pediatría según la guía IDSA 2013. Se consideró sepsis a la presencia de signos y síntomas de respuesta inflamatoria sistémica en el curso de una infección con; fiebre 
o hipotermia (temperatura rectal $>38,5^{\circ} \mathrm{C} \mathrm{o}<35^{\circ} \mathrm{C}$ ), taquicardia (puede estar ausente en pacientes con hipotermia), y al menos uno de los siguientes: status mental alterado, hipoxemia o pulsos débiles ${ }^{4}$.

Se utilizaron variables estadísticas de distribución y posición que fueron procesadas con el programa STATA versión 13.

\section{Resultados}

Se incluyeron 20 pacientes con diagnóstico de $\mathrm{AH}$, representando una tasa de hospitalización de 35 cada 100.000 admisiones (IC 95\% 21-54). La mediana de edad fue de 5 años, con un rango etario que osciló entre los 9 días y 17 años. Dieciséis pacientes eran de sexo masculino (80\%); con una relación masculino/femenino de 4/1.

La mayoría, 15/20 (75\%), eran previamente sanos. Cuatro niños $(20 \%)$ tenían antecedentes quirúrgicos recientes, dos de ellos habían sido sometidos a drenajes de abscesos de piel y tejidos blandos, y otros dos a apendicectomías. Un niño de seis años tenía como antecedente una atresia de vías biliares con cirugía de Kasai al mes de vida.

La fiebre estuvo presente en todos los casos. Un $60 \%$ presentó un síndrome febril prolongado (mayor a 14 días). La mitad de los pacientes tuvo dolor abdominal y $20 \%$ dificultad respiratoria. Se constató pérdida de peso, náuseas/vómitos y omalgia en $10 \%$ de los casos. Cinco pacientes $(25 \%)$ presentaron manifestaciones clínicas de una sepsis al ingreso. La mediana de los síntomas desde el inicio del cuadro clínico al diagnóstico fue de 12 días (rango 1-33).

Al examen físico 9/20 (45\%) presentaron hepatomegalia. Todos los pacientes eran eutróficos (Tabla 1).

Catorce pacientes $(70 \%)$ tenían infecciones asociadas de otras localizaciones (Tabla 2).

En relación a los parámetros de laboratorio, $85 \%$ de los pacientes presentó leucocitosis al ingreso, dos pacientes tuvieron leucopenia. El 95\% tuvo una PCR elevada con una mediana de $160 \mathrm{mg} / \mathrm{L}$ (rango 6-314). Ocho pacientes (40\%) mostraron elevación de transaminasas hepáticas (Tabla 3).

La ecografía abdominal permitió el diagnóstico en 18/20 (90\%) pacientes, en los dos restantes fue mediante una tomografía computada (TC). El seguimiento se realizó en todos los casos por ecografía, y en 11 pacientes se realizó una TC previo a la finalización del tratamiento. En 13 niños (65\%) se constató afectación del lóbulo derecho, $12(60 \%)$ presentaron un absceso único.

En todos los casos se tomaron dos hemocultivos por paciente, pesquisándose una bacteriemia en tres de ellos (15\%). Además, se obtuvieron cultivos microbiológicos en 17 pacientes: 13 (76\%) directamente del absceso, tres del espacio pleural y uno de un foco articular asociado. En
12 de ellos (70\%), se obtuvo aislamiento microbiológico. El patógeno más frecuente fue $S$. aureus, aislado en 10 pacientes $(50 \%), K$. pneumoniae (1), E. coli (1) y $S$. viridans (1). En 7 casos (35\%) no se obtuvo aislamiento microbiológico.

En todos los pacientes se realizó un estudio inmu-

\begin{tabular}{|lcc|}
$\begin{array}{l}\text { Tabla 1. Manifestaciones clínicas de niños con absceso } \\
\text { hepático piógeno }\end{array}$ & n casos & $\%$ \\
\hline Signos y síntomas & 20 & 100 \\
\hline Fiebre & 12 & 60 \\
\hline Fiebre prolongada & 10 & 50 \\
\hline Dolor abdominal & 9 & 45 \\
\hline Hepatomegalia & 5 & 25 \\
\hline Sepsis & 4 & 20 \\
\hline Dificultad respiratoria & 2 & 10 \\
\hline Náuseas y vómitos & 2 & 10 \\
\hline Pérdida de peso & 2 & 10 \\
\hline Omalgia & & \\
\hline
\end{tabular}

Tabla 2. Infecciones asociadas al absceso hepático piógeno y cultivo microbiológico

Infección asociada

Piel y tejidos blandos

Neumonía/ empiema

Granuloma eosinofílico

Odontógena

Peritonitis post-apendicectomía

Ninguno. Criptogénicos

$\begin{array}{cc}\mathbf{n} \text { casos } & \mathbf{n} \text { aislados } \\ 6 & 4 \\ 4 & 4 \\ 1 & 1 \\ 1 & 1 \\ 2 & 1 \\ 6 & 2\end{array}$

Microorganismo

SARM-AC

SARM-AC

SARM-AC

Streptococcus viridans

Escherichia coli

(1) Klebsiella pneumoniae (1) SASM

SARM-AC: Staphylococcus aureus resistente a meticilina adquirido en la comunidad. SASM: Staphylococcus aureus sensible a meticilina.

Tabla 3. Hallazgos de laboratorio

Medición
Leucocitos $\left(\mathrm{VN}: 5.300-11.500 / \mathrm{mm}^{3}\right)$
Leucocitosis $\left(>12.500 / \mathrm{mm}^{3}\right)$
Leucopenia $\left(<5.000 / \mathrm{mm}^{3}\right)$

PCR (V.N: 0-5 mg/L)

PCR elevada (> $10 \mathrm{mg} / \mathrm{L}$ )

$160(6-314)$

Mediana (rango) n casos (\%)

19.800 (3.700-39.900)

$17 / 20(85 \%)$

$2 / 20(10 \%)$

GPT (V.N: 5-33 U/L)

GPT elevada ( $>40 \mathrm{U} / \mathrm{L}$ )

$36(10-482)$

GOT (V.N: 5-32 U/L)

GOT elevada ( $>40 \mathrm{U} / \mathrm{L}$ )

$61(20-1147)$

VN: Valor de referencia. 


\begin{tabular}{|lcccl|}
\hline Tabla 4. Tratamiento antibacteriano empírico utilizado & y aislamiento microbiológico \\
\hline Fármacos & $\mathbf{n}$ casos & $\%$ & Adecuado & Motivo \\
Cefotaxima + clindamicina & 10 & $50 \%$ & Sí & \\
Meropenem + vancomicina & 3 & $15 \%$ & Sí & \\
Cefotaxima + vancomicina & 1 & $5 \%$ & Sí & \\
Piperacilina/tazobactam + vancomicina & 1 & $5 \%$ & Sí & \\
Vancomicina + cotrimoxazol & 1 & $5 \%$ & Sí & \\
Ampicilina + metronidazol + gentamicina & 1 & $5 \%$ & No & E. coli resistente \\
Ampicilina + gentamicina & 1 & $5 \%$ & No & SARM \\
Cefotaxima & 1 & $5 \%$ & No & SARM \\
Clindamicina & 1 & $5 \%$ & No & SARM resistente \\
\hline SARM: Staphylococcus aureus resistente a meticilina. & & & \\
\hline
\end{tabular}
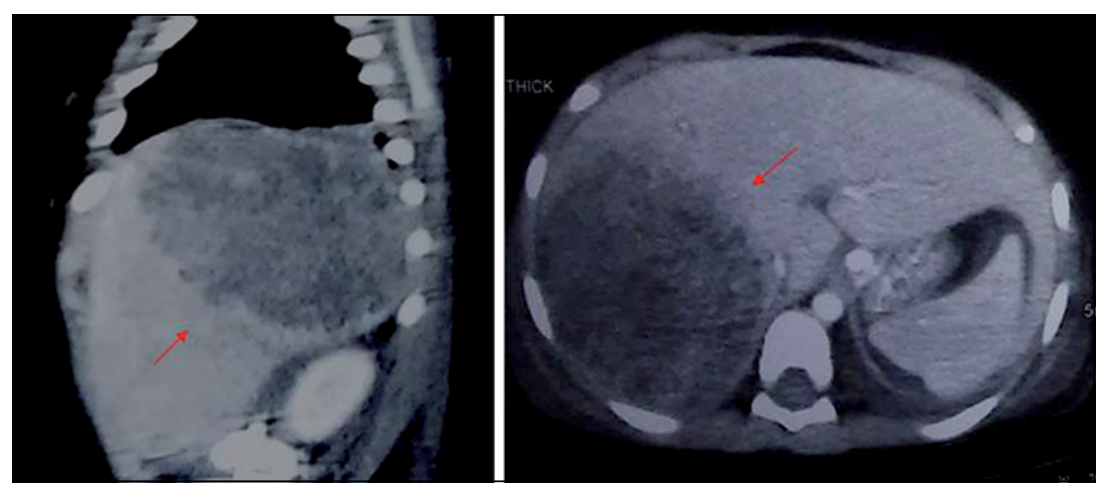

Figura 1. Tomografía computada (TC) de abdomen. Corte transversal y sagital. En hígado, imagen hipodensa, heterogénea. Absceso hepático por SARM-AC.

nológico con determinación de inmunoglobulinas, complemento, poblaciones linfocitarias y niveles de dihidrorodamina (DHR) que resultaron normales. Se realizó estudio parasitológico en deposiciones a 10 pacientes. En tres casos el resultado fue positivo, aislándose Giardia lamblia, Dientamoeba fragilis y Microsporidium sp. respectivamente, no asociados al desarrollo de $\mathrm{AH}$.

Se realizó drenaje quirúrgico en 13 pacientes $(65 \%)$, seis por punción percutánea, seis por cirugía a cielo abierto y en un caso por ambas técnicas.

Como tratamiento empírico inicial recibieron antibacterianos activos contra $S$. aureus, anaerobios y bacilos gramnegativos entéricos en 14/20 pacientes (70\%). En cuatro casos la terapia inicial fue considerada no adecuada (Tabla 4).

La mediana de tratamiento antimicrobiano i.v. fue de 30 días (rango 9-80) y la mediana de tratamiento total de 53 días (rango 17-80).

El $25 \%$ de los pacientes $(5 / 20)$ presentaron compli- caciones relacionadas a la internación: reacción cutánea secundaria a fármacos (1), escara por decúbito prolongado (1), sepsis intrahospitalaria (2), infección urinaria por Candida albicans (1). Dos pacientes requirieron internación en terapia intensiva con asistencia respiratoria mecánica por presentar un compromiso respiratorio secundario a una neumonía estafilocóccica. Todos los pacientes fueron controlados en el policlínico de Infectología hasta la resolución total del cuadro.

\section{Discusión}

El AH piógeno es infrecuente en pediatría. En Argentina son escasas las publicaciones referidas a esta patología. $\mathrm{Su}$ incidencia es mayor en países en vías de desarrollo como Brasil que publicó una incidencia de 1/138 admisiones e India con 79/100.000 admisiones. Esta cifra es ostensiblemente alta cuando se la compara con nuestros datos y aquellos reportados en países desarrollados como E.U.A con 25/100.000 admisiones o Dinamarca con $11 / 100.000$ admisiones $^{5-7}$.

Se describe la predisposición de desarrollar la enfermedad en niños con malnutrición y/o parasitosis; sin embargo, en este estudio, todos los niños fueron eutróficos y sólo tres de los diez estudiados presentaron parasitosis asociadas ${ }^{1}$. En ocasiones, la aparición de un AH piógeno puede señalar la presencia de una inmunodeficiencia subyacente ${ }^{1,5}$. En esta serie, en ningún paciente se demostró alteración de la inmunidad. Un hallazgo interesante es que la cuarta parte de ellos tuvieron factores predisponentes.

Los $\mathrm{AH}$ piógenos se presentan en forma variable dependiendo del mecanismo de infección. En esta serie, seis pacientes tuvieron un $\mathrm{AH}$ clasificado como primario o criptogénico; en el resto se encontró un foco infeccioso asociado. En cuatro casos, el AH se presentó concomitantemente con una neumonía con derrame paraneumónico; hallazgo que fue interpretado como una neumonía bacteriémica secundaria al AH. En estos cuatro casos el patógeno aislado fue $S$. aureus resistente a meticilina (SARM). En otros cuatro pacientes se presentaron con infecciones de piel y tejidos blandos al momento del diagnóstico, en los que también se aisló SARM.

Los niños en que se aisló SARM no presentaban patologías que condicionaran la concurrencia a un centro de salud ni presentaban internaciones recientes, por lo que se interpretan dichas infecciones como adquiridas en la comunidad (SARM-AC). Sin embargo, dos niños requirieron drenaje de abscesos subcutáneos una semana previa al diagnóstico del AH. En estos, si bien no puede descartarse completamente la colonización por microorganismos hospitalarios, teniendo en cuenta que la prevalencia de SARM-AC en nuestro medio es de $65 \%$, asumimos una infección de origen comunitario ${ }^{8}$. 
Los síntomas más frecuentes del $\mathrm{AH}$ piógeno son la fiebre y el dolor abdominal y menos prevalentes, los vómitos y la dificultad respiratoria ${ }^{1,2}$. En nuestra revisión, todos los niños presentaron fiebre y en la mitad de ellos se objetivó dolor abdominal. Cinco pacientes (25\%) presentaron sepsis, rescatándose en tres de ellos un patógeno responsable en los hemocultivos: K. pneumoniae en un neonato y SARM en dos pacientes con impactos múltiples.

La epidemiología en nuestro estudio mostró como patógeno predominante a $S$. aureus, similar a lo publicado tanto en países desarrollados como en Espíritu Santo, Brasil ${ }^{6,9}$. Yu-Lung Hsu publicó que, K. pneumoniae y Streptococcus spp. fueron los agentes etiológicos más frecuentes en Taiwan ${ }^{10}$.

Una alta proporción de niños presentan un síndrome febril prolongado como única manifestación ${ }^{1,2}$. En nuestro estudio, $60 \%$ presentó un síndrome febril por más de 14 días, sin otros síntomas. El promedio del período transcurrido desde el inicio de los síntomas hasta el diagnóstico fue de 12 días.

El 45\% presentó hepatomegalia, signo que orientaría al diagnóstico, así como la leucocitosis y el aumento de la PCR. En esta serie, al igual que en la literatura especializada, el aumento de las enzimas hepáticas se evidenció sólo en $40 \%$ de los casos.

Según distintos autores, la localización más frecuente se evidencia en el lóbulo hepático derecho, siendo habitualmente únicos ${ }^{1,2,11}$. En nuestra serie, $60 \%$ fueron únicos y $65 \%$ en el lóbulo derecho.

La ecografía presenta una sensibilidad de $81-100 \%$ considerando la condición de operador dependiente. La TC muestra una sensibilidad superior a $95-100 \%$ y podría detectar abscesos de hasta $1 \mathrm{~cm}$ de diámetro ${ }^{1,2,11}$. En nuestro estudio, en 18 pacientes el diagnóstico se realizó por ecografía, y en dos de ellos fue necesario realizar TAC.

La mayoría de los autores aconsejan el seguimiento sólo por ecografía para evitar la radiación ${ }^{11}$. El seguimiento evolutivo fue por este método en la totalidad de los pacientes. Se realizó TC al final del tratamiento a 11 pacientes $(55 \%)$ en nuestro estudio.

Los hemocultivos presentan una baja tasa de aislamiento microbiológico ${ }^{1}$. En nuestra serie, sólo tres pacientes tuvieron hemocultivos positivos, con clínica de sepsis al momento de la obtención de la muestra. En referencia a la edad, dos eran menores de 30 días de vida, uno con cultivo positivo a $K$. pneumoniae y SARM en otro; este último tenía además compromiso cutáneo, pulmonar y osteoarticular. El tercer paciente, de 6 años, presentaba el antecedente de una cirugía de Kasai al mes de vida, y desarrolló a una neumonía con supuración pleuropulmonar, con aislamiento de SARM en hemocultivos.

La punción guiada por ecografía o TC resulta una bue- na opción para precisar el diagnóstico y tratamiento ${ }^{1,11}$. Se realizó en seis pacientes en nuestro estudio, obteniendo un cultivo positivo sólo en uno de los seis casos. Asimismo, se obtuvo rescate microbiológico en los seis pacientes en que se realizó drenaje quirúrgico. En un paciente se realizaron ambas técnicas obteniendo aislamiento de microorganismos en ambos procedimientos. El drenaje quirúrgico a cielo abierto resultó ser la técnica más efectiva en cuanto al rendimiento microbiológico.

Los cultivos de sangre y material purulento fueron positivos en 13 pacientes (65\%), lo que permitió la indicación de antibioticoterapia adecuada. Coincidiendo con la literatura especializada, $S$. aureus fue el patógeno más frecuente, predominando en niños con infección de piel y tejidos blandos o respiratoria asociada ${ }^{9,11,12}$.

Para abscesos pequeños menores a $3 \mathrm{~cm}$ o múltiples el tratamiento puede basarse sólo en terapia antibacteriana. El drenaje por punción-aspiración, guiada por imágenes o la cirugía a cielo abierto es el tratamiento recomendado para casos de mayor magnitud. La elección entre punción-aspiración o cirugía a cielo abierto dependerá de la localización del absceso, tamaño y experiencia del equipo quirúrgico ${ }^{1,2,11}$. En nuestra serie 13 pacientes se sometieron a drenaje quirúrgico, seis con cirugía a cielo abierto, seis con punción-aspiración y uno por ambas técnicas. La elección de la técnica fue decisión del equipo quirúrgico.

El tratamiento empírico recomendado se basa en la cobertura de $S$. aureus y microorganismos del tracto digestivo (bacilos gramnegativos y anaerobios) ${ }^{13,14}$. En nuestra serie, cuatro pacientes no recibieron tratamiento empírico adecuado según el agente y sensibilidad antibacteriana (Tabla 4 ).

La duración del tratamiento antimicrobiano será menor si se realiza drenaje quirúrgico, alrededor de 2-3 semanas de tratamiento post procedimiento. Sin drenaje se puede prolongar por 4-6 semanas ${ }^{1,2,13}$. En este estudio, todos los pacientes recibieron antibioticoterapia por más de cuatro semanas (mediana 53 días total).

En conclusión, debería sospecharse $\mathrm{AH}$ piógeno en niños con síndrome febril prolongado de origen desconocido, leucocitosis y PCR elevada, especialmente si se asocia dolor abdominal, hepatomegalia o aumento de las enzimas hepáticas.

Los AH suelen ser oligosintomáticos, razón por la cual puede retrasarse el diagnóstico.

El principal agente etiológico en nuestra casuística fue SARM-AC, especialmente en niños previamente sanos con historia de infección de piel y tejidos blandos o respiratoria asociada.

Agradecimientos. Al Servicio de Microbiologia del Hospital de Niños "Dr. R. Gutiérrez": Bioquímicas M. Nancy Orlando, Adriana Procopio, Miryam Vázquez. 


\section{Resumen}

Introducción: El absceso hepático es una patología infrecuente en pediatría. Objetivo y Métodos: Describir las características de 20 pacientes ingresados entre 20092015 en el Hospital de Niños "R. Gutiérrez", Centro de Referencia de Nivel Terciario de Buenos Aires, Argentina con diagnóstico de absceso hepático. Resultados: La tasa de hospitalización fue 35/100.000 admisiones. La mediana de edad fue 5 años, relación masculino/femenino: 4/1. Cinco pacientes tuvieron factores predisponentes. La mediana de duración de la enfermedad al diagnóstico fue de 12 días. Todos los pacientes presentaron fiebre, 60\% síndrome febril prolongado y $50 \%$ dolor abdominal. La mayoría tenía leucocitosis y PCR elevada (mediana 160 $\mathrm{mg} / \mathrm{L}$ ). Sólo $40 \%$ tuvo alteración de pruebas hepáticas. En $90 \%$ de los casos el diagnóstico se realizó por ecografía, $65 \%$ estaban localizados en el lóbulo derecho; $60 \%$ eran lesiones únicas. Hubo aislamiento microbiológico en $12 / 17(70 \%)$ y en $3 / 20$ (15\%) de hemocultivos. Todos recibieron tratamiento antibacteriano empírico de amplio espectro. La mediana de tratamiento antibacteriano intravenoso fue 30 días y en total 53 días. Se realizó drenaje quirúrgico en 13 casos. Todos evolucionaron favorablemente. Conclusión: Staphylococcus aureus resistente a meticilina de la comunidad (SARM-AC) fue el patógeno predominante, especialmente en niños previamente sanos asociados a un foco cutáneo o respiratorio.

\section{Referencias bibliográficas}

1.- Brodley J S. Intra-abdominal, visceral and retroperitoneal abscesses. Principles and Practice of Pediatric Infectious Diseases. 2nd edition. Long S S, Pickering L K, Prober C G, eds. Elsevier. Philadelphia 2003, p 418-21.

2.- Sifri C D, Madoff L C. Chapter 72: Infections of the Liver and Biliary System. 2010. Mandell G L, Bennett J E, Dolin R. Eds. Mandell, Douglas, and Bennett's Principles of Practices of Infectious Diseases, $7^{\text {th }}$ Ed, Philadelphia: Churchill Livingstone Elsevier 2010; p 103542.

3.- National Committee for Clinical Laboratory Standards. Performance Standards for Antimicrobial Disk Suceptibility Test: Approved Standard, $7^{\text {th }}$ ed. NCCLS document M2-A7, vol 10, $n^{\circ}$ 1. Villanova, PA: National Committee for Clinical Laboratory Standards, 2000.

4.- Dellinger R P, Levy M M, Rhodes A,
Annane D, Gerlach H, Opal S M. Surviving sepsis campaign: international guidelines for management of severe sepsis and septic shock: 2012. Crit Care Med 2013; 41: 580-630.

5.- Pineiro-Carrero V M, Andres J M. Morbidity and mortality in children with pyogenic liver abscess. Am J Dis Child 1989; 143: 1424-7.

6.- Ferreira M A, Pereira F E, Musso C, Dettogni $R$ V. Pyogenic liver abscess in children: some observations in the Espírito Santo State, Brazil. Arq Gastroenterol 1997; 34: 49-54.

7.- Sharma M P, Kumar A. Liver abscess in children. Indian J Pediatr 2006; 73: 813-7.

8.- Pérez G, Martiren S, Reijtman V, Romero R, Mastroianni A, Casimir L, et al. Communityacquired Staphylococcus aureus bacteremia in children: a cohort study for 2010-2014. Arch Argent Pediatr 2016; 114: 508-13.

9.- Mishra K, Basu S, Roychoudhury S, Kumar P. Liver abscess in children: an overview. World $\mathrm{J}$ Pediatr 2010; 6: 210-6.

10.- Hsu Y L, Lin H C, Yen T Y, Hsieh T H, Wei H M, Hwang K P. Pyogenic liver abscess among children in a medical center in Central Taiwan. J Microbiol Immunol Infect 2015; 48: 302-5.

11.- Kaplan S L. Chapter 55. Pyogenic Liver Abscess. Feigin and Cherry's. Textbook of pediatric infectious diseases. Cherry J, Demmler-Harrison GJ, Kaplan S. Steinbach WJ, Hotez P. 6th edition. Saunders. Elsevier. 2009; p 689-92.

12.- Muorah M, Hinds R, Verma A, Yu D, Samyn M, Mieli-Vergani G, et al. Liver abscesses in children: a single center experience in the developed world. J Pediatr Gastroenterol Nutr 2006; 42: 201-6.

13.- Salahi R, Dehghani S M, Salahi H, Bahador A, Abbasy H R, Salahi F. Liver abscess in children: a 10-year single centre experience. Saudi J Gastroenterol 2011; 17: 199-202.

14.- Jiménez E, Tiberio G, Sánchez J, Jiménez F J, Jiménez G. Pyogenic hepatic abscesses: 16 years experience in its diagnosis and treatment. Enferm Infecc Microbiol Clin 1998; 16: 307-11. 